\title{
A new paradigm of quality of care in rheumatoid arthritis: how our new therapeutics have changed the game
}

\author{
Sonali P Desai ${ }^{*}$ and Daniel H Solomon ${ }^{2}$
}

\begin{abstract}
Demonstrating the effectiveness of expensive new rheumatoid arthritis (RA) therapeutics is imperative to determine whether the quality of care has improved with the introduction of these agents. Our current RA quality measures are primarily process based, but they must become outcomes based to better demonstrate quality. New RA quality measures must be multidimensional, accounting for all of the important outcomes in RA: radiographic, functional status, and disease activity. To fully understand the potential benefits of new therapeutics in RA, outcome measures must be integrated with routine practice.
\end{abstract}

New medications for rheumatoid arthritis (RA), combined with early, aggressive treatment strategies, have improved care. New biologic and small molecule therapies come with a hefty price tag, and demonstrating effectiveness is increasingly important: is the quality of care actually better for RA patients with newer therapies?

To answer this question, we must first define quality. As described by the Institute of Medicine, quality of care is 'the degree to which healthcare services for individuals and populations increase the likelihood of desired health outcomes and are consistent with current professional knowledge' [1]. Quality of care can be evaluated using quality measures, which are tools that provide the ability to quantify an aspect of healthcare relative to an established criterion [2]. Other facets of quality include patient satisfaction and access to care. Quality of care in RA is currently largely based on the use of processbased quality measures. For example: the frequency of

\footnotetext{
* Correspondence: sdesai5@partners.org

'Division of Rheumatology, Immunology, and Allergy, Brigham \& Women's

Hospital, 75 Francis Street, PBB-B3, Boston, MA 02115, USA

Full list of author information is available at the end of the article
}

disease-modifying anti-rheumatic drug (DMARD) prescribing in RA; the use of disease activity and functional status measures in routine practice; and laboratory monitoring frequency according to established recommendations. These RA quality measures are primarily derived from the Arthritis Foundation Starter Set and the Physician Quality Reporting Database RA measure set, but the American College of Rheumatology is actively developing a new RA measure set [3]. Although the current RA quality measures provide a reasonable starting point, they do not fully capture the spectrum of care quality for patients with RA in the United States.

Measures of quality of care are evolving to include concepts such as clinical outcomes. Some even argue that our primary goal should be to provide value: the health outcomes achieved per dollar spent [4]. This newer model incorporates the total cost of providing care to patients for a specific condition over a defined time period, relative to the health outcome achieved. For example, in RA the total cost of care would include nonbiologic and biologic DMARDs, office visits, physical therapy and inpatient hospitalizations. But the real question is how to best define outcomes in a chronic, complex condition such as RA? Outcomes can be multidimensional, accounting for all facets of care for a RA patient: radiographic progression, improvement in functional status score, or a decrease in disease activity score. Radiographic progression is often discussed as an important outcome in randomized controlled trials of RA therapeutics, but it is not a routine part of clinical practice. Measurement of functional status using a standardized, validated instrument is an important patientreported outcome, capturing key information about how RA impacts activities of daily living. Patient-reported outcomes are not used regularly in many busy, office practices despite the correlation with disease outcomes and mortality $[5,6]$. 
While professional groups such as the American College of Rheumatology have made recommendations on the measurement of disease activity through the use of tools such as the Disease Activity Score-28, the Clinical Disease Activity Index, or the Routine Assessment of Patient Disease Activity 3, documenting sustained low disease activity or remission requires multiple measurements [7]. Encouraging rheumatologists to treat to target and moving patients from high disease activity to remission is just one dimension of RA outcomes. Each potential clinical outcome has strengths and limitations and probably cannot serve as a standalone measure, but taken together they provide a more nuanced portrait of RA quality of care.

Moving from thinking about quality measures as process based to outcomes based is a significant challenge. To achieve good outcomes in RA using the new therapeutics in RA, one needs to consider the timing of therapy, the duration of treatment, and the co-existence of other medical conditions. Some patients may delay initiation of DMARD therapy due to fear of toxicity or lack of understanding of the risk/benefit profile; other patients may not be fully adherent to the treatment plan due to financial issues, socioeconomic factors or language barriers; and still others may not have access to rheumatology care until after they have sustained radiographic or functional damage from their RA. Since quality is often measured at the level of the rheumatologist, how do we risk adjust for these complex patient-related factors when evaluating outcomes in RA? Some rheumatologists see tertiary-care referral patients with longer disease duration, more treatment failures, and multiple co-morbidities. Developing appropriate case-mix adjustment tools to allow for meaningful comparison across providers is a huge task. We have learned that even for a simple quality measure such as whether RA patients receive a DMARD, this case-mix adjustment matters. When evaluating the quality measure on receipt of DMARDs for patients with RA, case-mix adjustment identified age, race and socioeconomic status as negative predictors of DMARD receipt [8].

Even though the road will be tough, we must determine how to best measure outcomes in RA to assess quality of care. The expenditures associated with biologic treatments raise important questions for how to demonstrate the effectiveness of medications for RA. However, there are emerging data on RA patients remaining in remission with fewer doses or even cessation of biologic drugs, raising the possibility that we can improve value for patients by simultaneously achieving good health outcomes and decreasing the overall cost of care [9]. An important first step to showing that new therapeutics are translating into better quality of care is incorporating the use of quantitative measurement of disease activity and functional status into routine clinical practice. By regularly measuring possible RA outcome measures, such as disease activity and functional status, we can identify patients who are achieving poor outcomes and create strategies to re-design care delivery for those patients. For example, the use of intensive nurse outreach between regularly scheduled rheumatologist visits to document medication adherence, side effects and education may improve outcomes faster and facilitate treating to target. Developing clinical riskadjustment tools for RA can help offset differences in patient case mix among rheumatologists.

However, measuring outcomes presents major challenges for the healthcare system in general. Collecting structured data to allow quality assessment is not routine in many practices and would place new burdens on the already stressed healthcare system, adding costs and frustration. Furthermore, accurately assessing quality of care requires adjusting for case-mix severity. This is especially true if outcomes become the focus of quality assessment. Collecting the dataset required for case-mix adjustment further taxes the healthcare provider. In addition, there are unanswered questions that remain: what is the current natural history of RA if diagnosed early and treated aggressively with combination nonbiologic and biologic DMARDs? To fully achieve the potential benefits of new therapeutics in RA, we first need RA quality measures that incorporate outcomes and these need to be easily integrated into typical practice.

\section{Abbreviations}

DMARD: Disease-modifying anti-rheumatic drug; RA: Rheumatoid arthritis.

\section{Competing interests}

The authors declare that they have no competing interests.

\section{Acknowledgements}

This work was supported by K24 AR 055989. DHS also receives salary support from research grants to Brigham and Women's Hospital from Lilly, Amgen, and CORRONA; serves in unpaid roles on trials sponsored by Pfizer and Novartis; and receives royalties from UpToDate.

\section{Author details}

'Division of Rheumatology, Immunology, and Allergy, Brigham \& Women's Hospital, 75 Francis Street, PBB-B3, Boston, MA 02115, USA. ²Division of Pharmacoepidemiology, Brigham \& Women's Hospital, 75 Francis Street, PBBB3, Boston, MA 02115, USA.

\section{Published: 29 Oct 2013}

\section{References}

1. Lohr KN: Medicare: A Strategy for Quality Assurance. Washington, DC: National Academy Press; 1990.

2. Desai SP, Yazdany J: Quality measurement and improvement in rheumatology: rheumatoid arthritis as a case study. Arthritis Rheum 2011, 63:3649-3660.

3. Khanna D, Arnold EL, Pencharz JN, Grossman JM, Traina SB, Lal A, MacLean $\mathrm{CH}$ : Measuring process of arthritis care: the Arthritis Foundation's quality indicator set for rheumatoid arthritis. Semin Arthritis Rheum 2006, 35:211237. 
4. Porter ME: What is value in health care? N Eng/ J Med 2010, 363:24772481.

5. Michaud K, Vera-Llonch M, Oster G: Mortality risk by functional status and health-related quality of life in patients with rheumatoid arthritis. J Rheumatol 2012, 39:54-59.

6. Castrejon I, Pincus T: Patient self-report outcomes to guide a treat-totarget strategy in clinical trials and usual clinical care of rheumatoid arthritis. Clin Exp Rheumatol 2012, 30(4 Suppl 73):S50-\$55.

7. Anderson J, Caplan L, Yazdany J, Robbins ML, Neogi T, Michaud K, Saag KG, O'Dell JR, Kazi S: Rheumatoid arthritis disease activity measures: American College of Rheumatology recommendations for use in clinical practice. Arthritis Care Res (Hoboken) 2012, 64:640-647.

8. Schmajuk G, Trivedi AN, Solomon DH, Yelin E, Trupin L, Chakravarty EF, Yazdany J: Receipt of disease-modifying antirheumatic drugs among patients with rheumatoid arthritis in Medicare managed care plans. JAMA 2011, 305:480-486.

9. Smolen JS, Nash P, Durez P, Hall S, llivanova E, Irazoque-Palazuelos F, Miranda P, Park MC, Pavelka K, Pedersen R, Szumski A, Hammond C, Koenig AS, Vlahos B: Maintenance, reduction, or withdrawal of etanercept after treatment with etanercept and methotrexate in patients with moderate rheumatoid arthritis (PRESERVE): a randomised controlled trial. Lancet 2013, 381:918-929.

\subsection{6/ar4356}

Cite this article as: Desai and Solomon: A new paradigm of quality of care in rheumatoid arthritis: how our new therapeutics have changed the game. Arthritis Research \& Therapy 2013, 15:121 